\title{
Ridge Fusion in Statistical Learning
}

\author{
Bradley S. Price, Charles J. Geyer, and Adam J. Rothman
}

\begin{abstract}
We propose a penalized likelihood method to jointly estimate multiple precision matrices for use in quadratic discriminant analysis and model based clustering. A ridge penalty and a ridge fusion penalty are used to introduce shrinkage and promote similarity between precision matrix estimates. Block-wise coordinate descent is used for optimization, and validation likelihood is used for tuning parameter selection. Our method is applied in quadratic discriminant analysis and semi-supervised model based clustering.
\end{abstract}

Key Words: Joint inverse covariance matrix estimation; Discriminant analysis; Model based clustering; Semi-supervised learning.

\section{INTRODUCTION}

Classification by quadratic discriminant analysis (QDA) requires the estimation of multiple inverse covariance matrices. In this model, the data $\left(x_{1}, y_{1}\right), \ldots,\left(x_{n}, y_{n}\right)$ are assumed to be a realization of $n$ independent copies of the random pair $(X, Y)$, where $Y$ is supported on $\mathcal{C}=\{1, \ldots, C\}$ and $(X \mid Y=c) \sim N_{p}\left(\mu_{0 c}, \Theta_{0 c}^{-1}\right)$ for each $c \in \mathcal{C}$. Let $n_{c}=\sum_{i=1}^{n} 1\left(y_{i}=c\right)$ be the sample size for the $c$ th class, let $\bar{x}_{c}=n_{c}^{-1} \sum_{i=1}^{n} x_{i} 1\left(y_{i}=c\right)$ be the observed sample mean for the $c$ th class, and let

$$
S_{c}=\frac{1}{n_{c}} \sum_{i=1}^{n}\left(x_{i}-\bar{x}_{c}\right)\left(x_{i}-\bar{x}_{c}\right)^{T} 1\left(y_{i}=c\right), \quad c \in \mathcal{C},
$$

Bradley S. Price, School of Statistics, University of Minnesota (E-Mail: price412@umn.edu). Charles J. Geyer, School of Statistics, University of Minnesota (E-mail: geyer@umn.edu). Adam J. Rothman, School of Statistics, University of Minnesota (E-mail: arothman@umn.edu). 
be the observed sample covariance matrix for the $c$ th class. Simply inverting $S_{c}$ to estimate $\Theta_{0 c}$ is problematic when $n_{c}$ is small and impossible when $p \geq n_{c}$. Pourahmadi (2011) reviews several regularized covariance and inverse covariance estimators that could be used to estimate the $\Theta_{0 c}$ 's, but this would not exploit similarities between them. Similarity between the $\Theta_{0 c}^{-1}$ 's and low condition numbers for each $\Theta_{0 c}$ are exploited in regularized discriminant analysis (RDA) (Friedman, 1989), which estimates $\Theta_{0 c}$ by inverting a linear combination of $S_{c}$, the identity matrix, and the observed pooled sample covariance matrix.

Minus 2 times the profile log-likelihood function, profiling over mean and class probability parameters, is

$$
g\left(\Theta_{1}, \ldots, \Theta_{C}\right)=\sum_{c \in \mathcal{C}} n_{c}\left\{\operatorname{tr}\left(S_{c} \Theta_{c}\right)-\log \operatorname{det}\left(\Theta_{c}\right)\right\},
$$

where $\operatorname{tr}$ and det are the trace and determinant operators. In a more general setting, Guo et al. (2011) and Danaher et al. (2013) proposed estimates of $\Theta_{01}, \ldots, \Theta_{0 C}$ by minimizing (1) plus penalties that promote entry equivalence across the estimates of $\Theta_{01}, \ldots, \Theta_{0 C}$ and zero entries within estimates of the $\Theta_{0 c}$ 's. The penalty of Guo et al. (2011) promoted zero entry equivalence across the inverse covariance estimates and the penalty of Danaher et al. (2013), called Fused Graphical Lasso (FGL), promoted zero and non-zero entry equivalence across the inverse covariance estimates. This sparse regularization is aimed at estimating multiple Gaussian graphical models, but is another natural regularization for QDA.

We propose estimates of $\Theta_{01}, \ldots, \Theta_{0 C}$ that minimize $g$ plus ridge penalties to promote entry-wise similarity between the estimates of the inverse covariance matrices and entry shrinkage for each inverse covariance estimate, which is yet another natural regularization for QDA. Our simulations and data examples illustrate cases where our estimators perform competitively in QDA. We also apply our method and FGL to model-based clustering.

Let $|A|_{q}$ denote the $q$-norm of the vector formed from all the entries of the matrix $A$. Let $\mathbb{S}^{p}$ denote the set of symmetric $p \times p$ matrices, and let $\mathbb{S}_{+}^{p}$ the set of symmetric $p \times p$ positive 
definite matrices.

Computing our estimates relies on evaluating the function $Q(\cdot, \lambda): \mathbb{S}^{p} \rightarrow \mathbb{S}^{p}$ defined by

$$
Q(S, \lambda)=\underset{\Theta \in \mathbb{S}_{+}^{p}}{\arg \min }\left\{\operatorname{tr}(\Theta S)-\log \operatorname{det}(\Theta)+\lambda|\Theta|_{2}^{2} / 2\right\} .
$$

Witten and Tibshirani (2009) used the optimization in (2) in the context of covarianceregularized regression, where $S$ is an observed sample covariance matrix and $\lambda$ is a nonnegative tuning parameter. For $\lambda>0$, they derived the closed-form solution

$$
Q(S, \lambda)=\frac{1}{2 \lambda} V\left\{-D+\left(D^{2}+4 \lambda I\right)^{1 / 2}\right\} V^{T},
$$

where $S=V D V^{T}$ with $V$ orthogonal and $D$ diagonal. Iterative algorithms that evaluate $Q(\cdot, \lambda)$ include the Fused Graphical Lasso (FGL) algorithm of Danaher et al. (2013) and an iterative algorithm developed by Rothman and Forzani (2013) that solves a modified

version of (2) in which the term $\lambda|\Theta|_{2}^{2} / 2$ is replaced by $\lambda \sum_{i, j} m_{i j} \theta_{i j}^{2} / 2$, where the $m_{i j}$ 's are user-specified non-negative penalty weights.

\section{JOINT ESTIMATION WITH RIDGE FUSION}

\section{$2.1 \quad$ Method}

We propose the penalized likelihood inverse covariance estimates

$$
\left(\widehat{\Theta}_{1}, \ldots, \widehat{\Theta}_{C}\right)=\underset{\Theta_{c} \in \mathbb{S}_{+}^{p}, c \in \mathcal{C}}{\arg \min }\left\{g\left(\Theta_{1}, \ldots, \Theta_{C}\right)+\frac{\lambda_{1}}{2} \sum_{c \in \mathcal{C}}\left|\Theta_{c}\right|_{2}^{2}+\frac{\lambda_{2}}{4} \sum_{(c, m) \in \mathcal{C} \times \mathcal{C}}\left|\Theta_{c}-\Theta_{m}\right|_{2}^{2}\right\},
$$

where $\lambda_{1}$ and $\lambda_{2}$ are non-negative tuning parameters. The term multiplied by $\lambda_{1}$ is called the ridge penalty, and the term multiplied by $\lambda_{2}$ is called the ridge fusion penalty. The former 
shrinks the elements of each $\widehat{\Theta}_{c}$ toward zero, and the latter promotes entry-wise similarity between $\widehat{\Theta}_{1}, \ldots, \widehat{\Theta}_{C}$. Although these estimates are not invariant to scaling of the variables, invariance is easily achieved by standardizing the variables and then rescaling appropriately. The objective function in (3) is strictly convex, and, if $\lambda_{1}>0$, then the global minimizer exists and is unique.

If $\lambda_{2}=0$, then (3) decouples into $C$ separate ridge penalized likelihood problems, which have solutions $\widehat{\Theta}_{c}=Q\left(S_{c}, n_{c}^{-1} \lambda_{1}\right)$ for $c \in \mathcal{C}$.

As $\lambda_{2}$ goes to infinity, $\left(\widehat{\Theta}_{1}, \ldots, \widehat{\Theta}_{C}\right)$ converges to $\left(\widehat{\Theta}_{1}^{\bullet}, \ldots, \widehat{\Theta}_{C}^{\bullet}\right)$ defined to be the solution to (3) subject to the constraint $\Theta_{1}=\cdots=\Theta_{C}$, which is

$$
\widehat{\Theta}_{1}^{\bullet}=\cdots=\widehat{\Theta}_{C}^{\bullet}=\underset{\Theta \in \mathbb{S}_{+}^{p}}{\arg \min }\left\{g(\Theta, \ldots, \Theta)+\frac{\lambda_{1}}{2} C|\Theta|_{2}^{2}\right\}=Q\left(\frac{1}{n} \sum_{c \in \mathcal{C}} n_{c} S_{c} ; \frac{\lambda_{1} C}{n}\right) .
$$

This "edge case" is important both for computational efficiency — solving (3) is computationally unstable when either $\lambda_{1}$ or $\lambda_{2}$ is very large due to the limited precision of computer arithmetic — and because it is itself a parsimonious model appropriate for some data.

\subsection{Algorithm}

We solve (3) using block-wise coordinate descent. The objective function in (3) is

$$
f\left(\Theta_{1}, \ldots, \Theta_{C}\right)=g\left(\Theta_{1}, \ldots, \Theta_{C}\right)+\frac{\lambda_{1}}{2} \sum_{c \in \mathcal{C}}\left|\Theta_{c}\right|_{2}^{2}+\frac{\lambda_{2}}{4} \sum_{(c, m) \in \mathcal{C} \times \mathcal{C}}\left|\Theta_{c}-\Theta_{m}\right|_{2}^{2}
$$

with $g$ defined by (1). The blockwise coordinate descent step minimizes this with respect to one $\Theta_{c}$, leaving the rest fixed. This step has a closed-form expression. Differentiating (5) with respect to $\Theta_{c}$ and setting the result equal to zero gives

$$
n_{c}\left(S_{c}-\Theta_{c}^{-1}\right)+\lambda_{1} \Theta_{c}+\lambda_{2} \sum_{m \in \mathcal{C} \backslash\{c\}}\left(\Theta_{c}-\Theta_{m}\right)=0
$$


and dividing through by $n_{c}$ gives

$$
\widetilde{S}_{c}-\Theta_{c}^{-1}+\tilde{\lambda}_{c} \Theta_{c}=0
$$

where

$$
\begin{aligned}
& \widetilde{S}_{c}=S_{c}-\frac{\lambda_{2}}{n_{c}} \sum_{m \in \mathcal{C} \backslash\{c\}} \Theta_{m} \\
& \tilde{\lambda}_{c}=\frac{\lambda_{1}+\lambda_{2}(C-1)}{n_{c}}
\end{aligned}
$$

and, since the left-hand side of (6) is the same as the gradient of the objective function of (2) with $S$ replaced by $\tilde{S}_{c}$ and $\lambda$ replaced by $\tilde{\lambda}_{c}$, the solution to (6), considered as a function of $\Theta_{c}$ only, is $Q\left(\widetilde{S}_{c} ; \tilde{\lambda}_{c}\right)$.

Algorithm 1. Initialize a convergence tolerance $\varepsilon$ and $\Theta_{1}, \ldots, \Theta_{C}$.

Compute $\tilde{\lambda}_{1}, \ldots, \tilde{\lambda}_{C}$ using $7 \mathrm{~b}$.

repeat

for $c \in \mathcal{C}$

Compute $\widetilde{S}_{c}$ using 7a.

Set $\Theta_{c}^{\text {old }}=\Theta_{c}$

Set $\Theta_{c}:=Q\left(\widetilde{S}_{c} ; \tilde{\lambda}_{c}\right)$.

end for

until

$$
\sum_{c \in \mathcal{C}}\left|\Theta_{c}^{\text {old }}-\Theta_{c}\right|_{1}<\varepsilon \sum_{c \in \mathcal{C}}\left|\left(S_{c} \circ I\right)^{-1}\right|_{1}
$$

\section{end repeat}

The computational complexity of the blockwise descent algorithm is $O\left(C p^{3}\right)$. The initial iterate for Algorithm 1 could be selected depending on the size of $\lambda_{2}$ : when $\lambda_{2}$ is large, one 
could initialize at the edge-case estimates defined in (4); and when $\lambda_{2}$ is small, one could initialize at the solution to (3) when $\lambda_{2}=0$.

\subsection{Tuning Parameter Selection}

Tuning parameter selection for (3) is done using a validation likelihood. This is a generalization of its use in the single precision matrix estimation problem (Huang et al., 2006, Rothman and Forzani, 2013). Randomly split the data into $K$ subsets, dividing each of the $C$ classes as evenly as possible. Let the subscript $(v)$ index objects defined for the $v$ th subset of the data, and $(-v)$ index those defined for the data with the $v$ th subset removed. The validation likelihood score is

$$
V\left(\lambda_{1}, \lambda_{2}\right)=\sum_{v=1}^{K} \sum_{c \in \mathcal{C}} n_{c(v)}\left\{\operatorname{tr}\left(S_{c(v)} \widehat{\Theta}_{c(-v)}\right)-\log \operatorname{det}\left(\widehat{\Theta}_{c(-v)}\right)\right\}
$$

noting that $\widehat{\Theta}_{c(-v)}$ depends on $\lambda_{1}$ and $\lambda_{2}$ even though the notation does not indicate this. Our selected tuning parameters are $\hat{\lambda}_{1}$ and $\hat{\lambda}_{2}$ defined as the values of the tuning parameters that minimize (8) over the set of their allowed values.

\section{DIFFERENCES BETWEEN RIDGE FUSION AND RDA}

To gain some understanding of the difference between our ridge fusion method and RDA, we consider the special case where there is no fusion. For RDA, this means that the coefficient multiplying the pooled sample covariance matrix is 0 , so its $c$ th covariance matrix estimate is $(1-\beta) S_{c}+\beta \bar{d} I$, where $\beta \in[0,1]$ is a tuning parameter and $\bar{d}$ is the arithmetic mean of the eigenvalues of $S_{c}$. Our ridge fusion method without fusion is defined by (3), with $\lambda_{2}=0$. Decompose $S_{c}=V D V^{T}$ with $V$ orthogonal and $D$ diagonal. The $c$ th covariance estimate 
for ridge fusion without fusion is

$$
V\left\{0.5 D+0.5\left(D^{2}+4 \lambda_{1} n_{c}^{-1} I\right)^{1 / 2}\right\} V^{T}
$$

and the $c$ th covariance estimate for RDA without fusion is $V\{(1-\beta) D+\beta \bar{d} I\} V^{T}$. Both estimates have the same eigenvectors as $S_{c}$, but their eigenvalues are different. RDA shrinks or inflates the eigenvalues of $S_{c}$ linearly toward their average $\bar{d}$. Ridge fusion inflates the eigenvalues of $S_{c}$ nonlinearly, where the smaller eigenvalues of $S_{c}$ are inflated more than the larger eigenvalues.

\section{EXTENSION TO SEMI-SUPERVISED MODEL BASED CLUSTERING}

\subsection{Introduction}

Just as in classification using QDA, semi-supervised model based clustering with Gaussian mixture models requires estimates for multiple inverse covariance matrices. In the semisupervised model, let $\mathcal{L}$ and $\mathcal{U}$ be disjoint sets of cardinality $n_{L}$ and $n_{U}$, respectively. The data are random pairs $\left(X_{i}, Y_{i}\right)$, where for $i \in \mathcal{L}$ both $X_{i}$ and $Y_{i}$ are observed but for $i \in \mathcal{U}$

only $X_{i}$ is observed ( $Y_{i}$ is latent). We denote $\mathcal{D}$ as the observed data. Otherwise, the setup is as in Section 2 .

Let the conditional probability density function of $X_{i}$ given $Y_{i}=c$, which, as in section 2 , we assume is Gaussian, be denoted by $\phi\left(\cdot ; \mu_{c}, \Theta_{c}\right)$, where $\mu_{c}$ is the mean vector and $\Theta_{c}$ is the inverse covariance matrix. Let $\pi_{c}$ denote the probability of $Y_{i}=c$. And let $\Psi=$ $\left\{\Theta_{1}, \ldots, \Theta_{c}, \mu_{1}, \ldots, \mu_{c}, \pi_{1}, \ldots, \pi_{c}\right\}$ denote all the parameters. 
The $\log$-likelihood for the observed data $\mathcal{D}$ with parameters $\Psi$ is

$$
l(\Psi)=\sum_{i \in \mathcal{L}} \log \left\{\pi_{y_{i}} \phi\left(x_{i} ; \mu_{y_{i}}, \Theta_{y_{i}}\right)\right\}+\sum_{i \in \mathcal{U}} \log \left\{\sum_{c \in \mathcal{C}} \pi_{c} \phi\left(x_{i} ; \mu_{c}, \Theta_{c}\right)\right\}
$$

and the complete data log-likelihood (treating the unobserved data as if it were observed) is

$$
h(\Psi)=\sum_{i \in \mathcal{L} \cup \mathcal{U}} \log \left\{\pi_{y_{i}} \phi\left(x_{i} ; \mu_{y_{i}}, \Theta_{y_{i}}\right)\right\} .
$$

Methods proposed in Ruan et al. (2011), Xie et al. (2008), and Zhou et al. (2009) seek to estimate the parameters of (9) using a penalized EM algorithm with assumptions of a specific structure or sparsity on both means and inverse covariances. We propose to estimate these parameters by maximizing (9) penalized by ridge or $l_{1}$ penalties to create the same kind of shrinkage discussed in sections 1 and 2. We also will address tuning parameter selection by introducing a validation likelihood that uses the unlabeled data.

\subsection{Joint Estimation In Semi-Supervised Model Based Clustering}

The penalized log likelihood is

$$
l(\Psi)-\frac{\lambda_{1}}{j} \sum_{c \in \mathcal{C}}\left|\Theta_{c}\right|_{j}^{j}-\frac{\lambda_{2}}{j^{2}} \sum_{(c, m) \in \mathcal{C} \times \mathcal{C}}\left|\Theta_{c}-\Theta_{m}\right|_{j}^{j}
$$

for $j \in\{1,2\}$. When $j=1$ (11) uses the Fused Graphical Lasso (FGL) penalty (Danaher et al. 2013), and when $j=2$ (11) uses the the ridge fusion method penalty of section 2. Here we are introducing these penalties to semi-supervised model based clustering.

We use the penalized analog of the EM Algorithm to find maximum penalized likelihood estimates of 11) Dempster et al., 1977; Wu, 1983; Green, 1990) . Let $\widehat{\Psi}$ denote the current 
iterate of the parameter estimates. Then the E-Step of the algorithm calculates

$$
\begin{aligned}
Q_{\widehat{\Psi}}(\Psi)= & E_{\widehat{\Psi}}\left(h(\Psi)-\frac{\lambda_{1}}{j} \sum_{c \in \mathcal{C}}\left|\Theta_{c}\right|_{j}^{j}-\frac{\lambda_{2}}{j^{2}} \sum_{(c, m) \in \mathcal{C} \times \mathcal{C}}\left|\Theta_{c}-\Theta_{m}\right|_{j}^{j} \mid \mathcal{D}\right), \\
= & \sum_{i \in \mathcal{L}} \log \left\{\pi_{y_{i}} \phi\left(x_{i} ; \mu_{y_{i}}, \Theta_{y_{i}}\right)\right\}+\sum_{i \in \mathcal{U}} \sum_{c \in \mathcal{C}} \alpha_{i c} \log \left\{\pi_{c} \phi\left(x_{i} ; \mu_{c}, \Theta_{c}\right)\right\} \\
& -\frac{\lambda_{1}}{j} \sum_{c \in \mathcal{C}}\left|\Theta_{c}\right|_{j}^{j}-\frac{\lambda_{2}}{j^{2}} \sum_{(c, m) \in \mathcal{C} \times \mathcal{C}}\left|\Theta_{c}-\Theta_{m}\right|_{j}^{j},
\end{aligned}
$$

where

$$
\alpha_{i c}=\frac{\phi\left(x_{i} ; \hat{\mu}_{c}, \hat{\Theta}_{c}\right) \hat{\pi}_{c}}{\sum_{m \in \mathcal{C}} \phi\left(x_{i} ; \hat{\mu}_{m}, \hat{\Theta}_{m}\right) \hat{\pi}_{m}}, \quad i \in \mathcal{U} \text { and } c \in \mathcal{C}
$$

The M-Step of the algorithm calculates $\widehat{\Psi}$ that maximizes (12) with respect to $\Psi$. Define

$$
\begin{gathered}
\tilde{n}_{c}=\sum_{i \in \mathcal{L}} 1\left(y_{i}=c\right)+\sum_{i \in \mathcal{U}} \alpha_{i c} \\
\tilde{\pi}_{c}=\frac{\tilde{n}_{c}}{n_{L}+n_{U}}, \\
\tilde{\mu}_{c}=\frac{\sum_{i \in \mathcal{L}} x_{i} 1\left(y_{i}=c\right)+\sum_{i \in \mathcal{U}} \alpha_{i c} x_{i}}{\tilde{n}_{c}} \\
\tilde{S}_{c}^{(L)}=\frac{\sum_{i \in \mathcal{L}} 1\left(y_{i}=c\right)\left(x_{i}-\tilde{\mu}_{c}\right)\left(x_{i}-\tilde{\mu}_{c}\right)^{T}}{n_{c}}, \\
\tilde{S}_{c}^{(U)}=\frac{\sum_{i \in \mathcal{U}} \alpha_{i c}\left(x_{i}-\tilde{\mu}_{c}\right)\left(x_{i}-\tilde{\mu}_{c}\right)^{T}}{\sum_{i \in \mathcal{U}} \alpha_{i c}}, \\
\tilde{S}_{c}=\frac{n_{c} \tilde{S}_{c}^{(L)}+\left(\sum_{i \in \mathcal{U}} \alpha_{i c}\right) \tilde{S}_{c}^{(U)}}{\tilde{n}_{c}} .
\end{gathered}
$$

Then the profile of the negative penalized complete data log-likelihood for the $\Theta$ 's replacing $\mu_{c}$ with $\tilde{\mu}_{c}$ and $\pi_{c}$ with $\tilde{\pi}_{c}$ is

$$
\sum_{c \in \mathcal{C}} \tilde{n}_{c}\left\{\operatorname{tr}\left(\tilde{S}_{c} \Theta_{c}\right)-\log \operatorname{det}\left(\Theta_{c}\right)\right\}+\frac{\lambda_{1}}{j} \sum_{c \in \mathcal{C}}\left|\Theta_{c}\right|_{j}^{j}+\frac{\lambda}{j^{2}} \sum_{(c, m) \in \mathcal{C} \times \mathcal{C}}\left|\Theta_{c}-\Theta_{m}\right|_{j}^{j},
$$

and maximizing this subject to $\Theta_{c} \in \mathbb{S}_{+}^{p}$ gives estimates of the $\Theta$ 's for the next iteration, 
estimates of the other parameters for the next iteration being given by (14) and (15). In the

$j=1$ case of (16) solutions are found by the FGL algorithm (Danaher et al., 2013), and in the $j=2$ case solutions are found by our coordinate descent algorithm (Algorithm 1 ). In our current implementation, both algorithms are run until convergence.

All of the steps above are repeated until the penalized EM (PEM) algorithm converges. Our convergence criterion here is similar to the one in Section 2.2, in particular, we are using the difference in the $\alpha$ 's from iteration to iteration. Green (1990) gives convergence rates for the penalized EM algorithm that vary with the proportion of unlabeled data (the more unlabeled data the worse the convergence). Thus PEM should work well when the proportion of the unlabeled data is not too large. The initial estimates for our EM algorithm are obtained from the labeled data (Basu et al., 2002).

An alternative is to not iterate to convergence in the optimization of (16). Dempster et al. (1977) call a variant of the EM algorithm in which the M-step is not iterated to convergence but does make progress (goes uphill on the function it is optimizing) a generalized EM (GEM) algorithm and $\mathrm{Wu}(1983)$ proves this also converges to the MLE (under certain conditions). The analog here, not iterating the M-step to convergence, is penalized generalized EM (PGEM) and should also converge to the penalized maximum likelihood estimate, although we have not investigated this.

\subsection{Validation Likelihood for Tuning Parameter Selection}

In the semi-supervised setting it is not uncommon to have data in which the labeled sample size for each class is so small that it would not be practical to use the validation likelihood presented in section 2.3 to select the tuning parameters. To address this we propose a validation likelihood that uses both labeled and unlabeled data. The negative log-likelihood 
of the observed data $\mathcal{D}$ with parameters $\Psi$ is

$$
L_{\mathcal{D}}(\Psi)=-\sum_{i \in \mathcal{L}} \log \left\{\pi_{y_{i}} \phi\left(x_{i} ; \mu_{y_{i}}, \Theta_{y_{i}}\right)\right\}-\sum_{i \in \mathcal{U}} \log \left\{\sum_{c \in \mathcal{C}} \pi_{c} \phi\left(x_{i} ; \mu_{c}, \Theta_{c}\right)\right\} .
$$

Similar to the supervised case, randomly split the labeled and unlabeled data into $K$ subsets. We define $\mathcal{L}_{(v)}$ and $\mathcal{U}_{(v)}$ to be the indices of the $v$ th subset of the labeled and unlabeled data and $\mathcal{D}_{(v)}$ to be the $v$ th subset of the data. Let $\widehat{\Psi}_{(-v)}$ denote the parameter estimates resulting from the semi-supervised model based clustering on the data with $\mathcal{D}_{(v)}$ removed.

The validation likelihood is $L_{\mathcal{D}_{(v)}}\left(\widehat{\Psi}_{(-v)}\right)$, which is the negative log-likelihood for the $v$ th subset of the data with parameters estimates derived from all the data except that subset The validation score is

$$
V\left(\lambda_{1}, \lambda_{2}\right)=\sum_{v=1}^{K} L_{\mathcal{D}_{(v)}}\left(\widehat{\Psi}_{(-v)}\right)
$$

where $\widehat{\Psi}_{(-v)}$ are the parameter estimates based on $\lambda_{1}$ and $\lambda_{2}$ though the notation does not say this specifically. We select the tuning parameters $\hat{\lambda}_{1}$ and $\hat{\lambda}_{2}$ that minimize (18) over the set of allowed tuning parameter values.

\section{SIMULATIONS}

\subsection{Regularization in quadratic discriminant analysis}

We present simulation studies that compare the classification performance of QDA in which RDA, FGL, and the ridge fusion methods are used to estimate the inverse covariance matrices. 


\subsubsection{The data generating model and performance measurements}

In the following simulations described in sections 5.1.2 -5.1.4, we generated data from a two-class model where the class 1 distribution was $N_{p}\left(\mu_{1}, \Sigma_{1}\right)$ and the class 2 distribution was $N_{p}\left(\mu_{2}, \Sigma_{2}\right)$. We considered $p=50$ and $p=100$. The training data had 25 independent draws from the class 1 distribution and 25 independent draws from the class 2 distribution. These training observations were used to compute parameter estimates. These estimates were used in QDA to classify observations in an independent testing dataset consisting of 500 independent draws from the class 1 distribution and 500 independent draws from the class 2 distribution. We measured performance with the classification error rate (CER) on these testing cases. This process was replicated 100 times.

The tuning parameters $\lambda_{1}$ and $\lambda_{2}$ for FGL and the ridge fusion estimates of $\Sigma_{1}^{-1}$ and $\Sigma_{2}^{-1}$ were selected from a subset of $\left\{10^{x}: x=-10,-9.5, \ldots, 9,9.5,10\right\}$ using the method described in section 2.3 unless otherwise stated. Specific subsets were determined from pilot tests for each simulation. An R package, RidgeFusion, implementing the ridge fusion and tuning parameter selection methods is available on CRAN (Price, 2014).

\subsubsection{RDA tuning parameter selection simulation}

In this simulation, we compared two cross-validation procedures to select tuning parameters for the RDA estimators of $\Sigma_{1}^{-1}$ and $\Sigma_{2}^{-1}$. The first procedure minimizes the validation CER and the second maximizes the validation likelihood, as described in section 2.3. Weihs et al. (2005), in the documentation of the $\mathrm{R}$ package $\mathrm{klaR}$, mentioned that cross validation minimizing validation CER is unstable when sample sizes are small. We used the klaR package to perform RDA with tuning parameter selection that minimizes validation CER, and we used our own code to perform RDA with tuning parameter selection that maximizes validation likelihood.

We set all elements of $\mu_{1}$ to $5 p^{-1} \log (p)$ and made $\mu_{2}$ the vector of zeros. We generated 
Table 1: Average CER for RDA reported with standard errors based on 100 independent replications for the simulation described in section 5.1.2 (the RDA tuning parameter selection simulation).

\begin{tabular}{l|ccc} 
& $p=20$ & $p=50$ & $p=100$ \\
\hline Validation Likelihood & $0.02(0.01)$ & $0.05(0.02)$ & $0.13(0.04)$ \\
Cross Validation with CER & $0.07(0.03)$ & $0.09(0.03)$ & $0.13(0.04)$
\end{tabular}

$\Sigma_{1}$ and $\Sigma_{2}$ to have the same eigenvectors, which were the right singular vectors of the 100 by $p$ matrix with rows independently drawn from $N_{p}(0, I)$. The $j$ th eigenvalue of $\Sigma_{1}$ is

$$
100 \frac{p-j+1}{p} \mathrm{I}\{1 \leq j \leq 6\}+10 \frac{p-j+1}{p} \mathrm{I}\{7 \leq j \leq 11\}+\frac{p-j+1}{p} \mathrm{I}\{12 \leq j \leq p\} .
$$

The $j$ th eigenvalue of $\Sigma_{2}$ is

$$
500 \frac{p-j+1}{p} \mathrm{I}\{1 \leq j \leq 6\}+50 \frac{p-j+1}{p} \mathrm{I}\{7 \leq j \leq 11\}+\frac{p-j+1}{p} \mathrm{I}\{12 \leq j \leq p\} .
$$

We investigated cases where $p=20,50,100$. The results of this simulation, found in Table 1 , indicate that cross validation maximizing validation likelihood outperforms cross validation minimizing CER. This lead us to tune RDA with the validation likelihood method in the remaining simulation studies.

\subsubsection{Dense, ill conditioned, and unequal inverse covariance matrices simula- tion: part 1}

This simulation uses the parameter values described in section 5.1 .2 to compare the QDA classification performance of FGL, RDA, and the ridge fusion methods. Since $\Sigma_{1}^{-1}$ and $\Sigma_{2}^{-1}$ are dense, it is unclear which method should perform the best. Based on section 3, we expect that RDA will perform poorly because $\Sigma_{1}$ and $\Sigma_{2}$ are ill conditioned. Table 2 has the average CER and corresponding standard errors. The ridge fusion method outperforms RDA. We 
Table 2: Average CER for QDA with standard errors based on 100 independent replications for the simulation described in section 5.1 .3 (the dense, ill conditioned, and unequal inverse covariance matrices simulation: part 1).

\begin{tabular}{l|cc} 
& $p=50$ & $p=100$ \\
\hline RDA & $0.05(0.02)$ & $0.13(0.04)$ \\
Ridge & $0.03(0.02)$ & $0.08(0.03)$ \\
FGL & $0.03(0.02)$ & $0.09(0.02)$
\end{tabular}

Table 3: Average CER for QDA reported with standard errors based on 100 replications for the simulation described in section 5.1.4 (the dense, ill conditioned, and unequal inverse covariance matrices simulation: part 2).

\begin{tabular}{c|c|c} 
& $p=50$ & $p=100$ \\
\hline Ridge & $0.00(0.00)$ & $0.00(0.00)$ \\
RDA & $0.16(0.04)$ & $0.31(0.05)$ \\
FGL & $0.00(0.00)$ & $0.00(0.00)$
\end{tabular}

also see that ridge fusion and FGL perform similarly.

\subsubsection{Dense, ill conditioned, and unequal inverse covariance matrices simula- tion: part 2}

In this simulation, $\Sigma_{1}$ has $(i, j)$ th entry $0.5 \cdot 1(|i-j|=1)+1(i=j)$, and $\Sigma_{2}$ is defined in section 5.1.2. We set each element in $\mu_{1}$ to $p^{-1}$ and each element of $\mu_{2}$ to be zero. We expect RDA to perform poorly because of the large condition numbers and lack of similarity between $\Sigma_{1}$ and $\Sigma_{2}$. The average classification error rate is reported in Table 3, where we see that ridge fusion and FGL outperform RDA for both values of $p$.

\subsubsection{Sparse, well conditioned, and equal inverse covariance matrices simulation}

In this simulation we set $\Sigma_{1}=\Sigma_{2}=I$ and all elements of $\mu_{1}$ to $10 p^{-1} \log (p)$ and all elements of $\mu_{2}$ to zero. The average CER, based on 100 replications, is reported in Table 4: all three 
Table 4: Average CER for QDA reported with standard errors based on 100 independent replications for the simulation described in section 5.1.5 (the sparse, well conditioned, and equal inverse covariance matrices simulation).

\begin{tabular}{l|cc} 
& $p=50$ & $p=100$ \\
\hline RDA & $0.01(0.01)$ & $0.03(0.02)$ \\
Ridge & $0.01(0.01)$ & $0.04(0.02)$ \\
FGL & $0.01(0.01)$ & $0.03(0.02)$
\end{tabular}

methods perform similarly when $p=50$ and the ridge fusion method is outperformed by RDA and FGL when $p=100$.

\subsubsection{Sparse and similar inverse covariance matrices simulation}

In this simulation, $\Sigma_{1}$ is block diagonal with two equal size blocks: the $(i, j)$ th entry in the first block was $0.95^{|i-j|}$ and the $(k, m)$ th entry in the second block was $0.8^{|k-m|}$. We also made $\Sigma_{2}$ block diagonal with two equal size blocks: the $(i, j)$ th entry in the first block was $0.95^{|i-j|}$ and the $(k, m)$ th entry in the second block was $\rho^{|k-m|}$, where $\rho=0.25,0.50$, and 0.95. This setting should favor FGL, which exploits the sparsity in $\Sigma_{1}^{-1}$ and $\Sigma_{2}^{-1}$. We set each element in $\mu_{1}$ to $20 p^{-1} \log (p)$ and each element in $\mu_{2}$ to zero. The classification performance is reported in Table 5. We see that FGL outperforms the other two methods for $\rho=0.25,0.50$ and all values of $p$. When $\rho=0.95$, even though the covariance matrices are ill conditioned, RDA outperforms the ridge fusion method and FGL for both values of $p$.

\subsubsection{Inverse covariance matrices with small entries simulation}

In this simulation, $\Sigma_{1}$ has $(i, j)$ th entry $0.4 \cdot 1(|i-j|=1)+1(i=j)$ and $\Sigma_{2}$ has $(i, j)$ th entry $\rho \cdot 1(|i-j|=1)+1(i=j)$, where $\rho=0.25,0.30,0.35$ and 0.50 . We set each element in $\mu_{1}$ to $10 \log (p) p^{-1}$ and each element in $\mu_{2}$ to zero. The classification results are reported in Table 6, and show that RDA has the best classification performance for each value of $p$ and 
Table 5: Average CER for QDA reported with standard errors based on 100 independent replications for the simulation described in section 5.1.6 (the sparse and similar inverse covariance matrices simulation).

\begin{tabular}{l|c|cc} 
& $\rho$ & $p=50$ & $p=100$ \\
\hline RDA & & $0.10(0.03)$ & $0.21(0.04)$ \\
Ridge & 0.95 & $0.13(0.04)$ & $0.24(0.04)$ \\
FGL & & $0.11(0.03)$ & $0.21(0.04)$ \\
\hline RDA & & $0.08(0.03)$ & $0.20(0.04)$ \\
Ridge & 0.50 & $0.06(0.02)$ & $0.13(0.04)$ \\
FGL & & $0.04(0.02)$ & $0.09(0.03)$ \\
\hline RDA & & $0.06(0.02)$ & $0.15(0.04)$ \\
Ridge & 0.25 & $0.05(0.02)$ & $0.12(0.03)$ \\
FGL & & $0.03(0.02)$ & $0.06(0.02)$
\end{tabular}

$\rho$. Note that FGL has the same average classification error rate as RDA in the case where $p=50$ when $\rho=0.30$ and 0.35 .

\subsection{Computing time simulations: ridge fusion versus FGL}

Although FGL performed as well or better than our ridge fusion method at classification in the simulations of sections 5.1.3 - 5.1.4, we found that computing FGL is much slower than our ridge fusion method when a dense estimate is desired. We present three timing simulations that illustrate this pattern. In each simulation we measured the computing time (in seconds) of ridge fusion and FGL, calculated by the $\mathrm{R}$ function system.time, where the tuning parameters $\left(\lambda_{1}, \lambda_{2}\right)$ are selected from $\Lambda \times \Lambda$, where $\Lambda=\left\{10^{x}: x=-8,-7, \ldots, 7,8\right\}$ and $p=100$. We report the average of the difference in computing time between ridge fusion and FGL based on 100 independent replications, for each point in $\Lambda \times \Lambda$. FGL and ridge fusion were computed using the JGL (Danaher, 2013) and RidgeFusion (Price, 2014) $R$ packages with default settings.

In each simulation setting, the ridge fusion algorithm is faster than FGL when $\lambda_{1}$ is small, 
Table 6: Average CER for QDA reported with standard errors based on 100 replications for the simulation described in section 5.1.7 (the inverse covariance matrices with small entries simulation).

\begin{tabular}{c|c|c|c} 
& $\rho$ & $p=50$ & $p=100$ \\
\hline Ridge & & $0.04(0.02)$ & $0.09(0.03)$ \\
RDA & 0.25 & $0.02(0.01)$ & $0.06(0.02)$ \\
FGL & & $0.03(0.02)$ & $0.07(0.03)$ \\
\hline Ridge & & $0.04(0.02)$ & $0.09(0.03)$ \\
RDA & 0.30 & $0.03(0.02)$ & $0.06(0.02)$ \\
FGL & & $0.03(0.02)$ & $0.08(0.03)$ \\
\hline Ridge & & $0.04(0.02)$ & $0.10(0.03)$ \\
RDA & 0.35 & $0.03(0.02)$ & $0.07(0.03)$ \\
FGL & & $0.03(0.02)$ & $0.08(0.03)$ \\
\hline Ridge & & $0.06(0.02)$ & $0.11(0.03)$ \\
RDA & 0.50 & $0.03(0.02)$ & $0.09(0.03)$ \\
FGL & & $0.04(0.02)$ & $0.10(0.03)$
\end{tabular}

and FGL is faster than ridge fusion when $\lambda_{1}$ is large. This result is not surprising as a large $\lambda_{1}$ when using FGL will produce sparse estimates of the inverse covariance matrices, which the algorithm exploits in estimation by using a divide and conquer algorithm. In summary, FGL will be faster when the true inverse covariance matrices are quite sparse and otherwise ridge fusion will be faster.

\subsubsection{Dense, ill conditioned, and different inverse covariance matrices timing simulation}

This simulation investigates the difference of the average speed over 100 replications of FGL and ridge fusion using the data generating model described in section 5.1.1 and parameter values used in section 5.1.3. The results are shown in in Figure 1. The ridge fusion method is faster or comparable to FGL when $\lambda_{1}$ is small and otherwise FGL is faster. Over the entire grid we find that, on average, ridge fusion is 4 seconds faster than FGL. At one grid point, ridge fusion was 534 times faster than FGL and at another grid point FGL was 73 


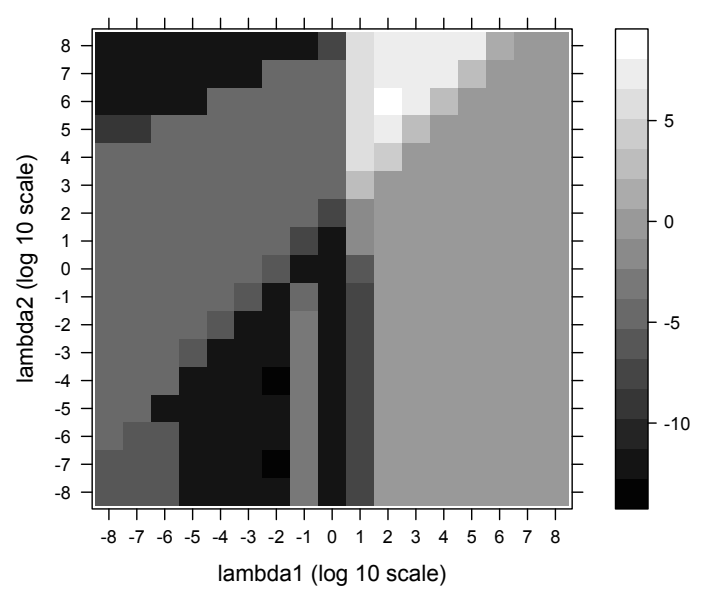

Figure 1: Difference of average computing time for the simulation described in section 5.2.1 based on 100 replications at each point in $\Lambda \times \Lambda$. Negative values represent where ridge fusion is faster than FGL.

times faster than ridge fusion.

\subsubsection{Sparse and similar inverse covariance matrices timing simulation}

This simulation uses the data generating model described in section 5.1.1 and parameter values used in section 5.1.6 with $\rho=0.95$ and $p=100$. Here FGL performs much better than ridge fusion in classification. The results shown in Figure 2 are the difference of the average computing time of FGL and ridge fusion. These are similar to those of section 5.2.1. As expected, ridge fusion is faster or comparable to FGL when $\lambda_{1}$ is small and otherwise FGL is faster. Averaging across the grid, ridge fusion was approximately 5 seconds faster. At the extremes, there was one grid point at which ridge fusion was 564 times faster than FGL, and another point at which FGL was 63 times faster than ridge fusion.

\subsubsection{Inverse covariance matrices with small entries timing simulation}

This simulation uses the data generating model described in section 5.1.1 and parameter values used in section 5.1 .7 when $\rho=0.50$ and $p=100$. The results in Figure 3 show a 


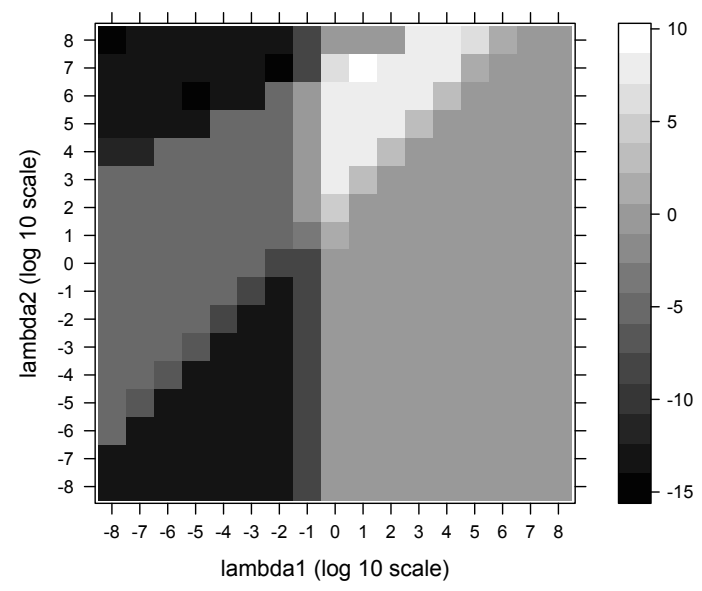

Figure 2: Difference of average computing time for the simulation described in section 5.2 .2 based on 100 replications at each point in $\Lambda \times \Lambda$. Negative values represent where ridge fusion is faster than FGL.

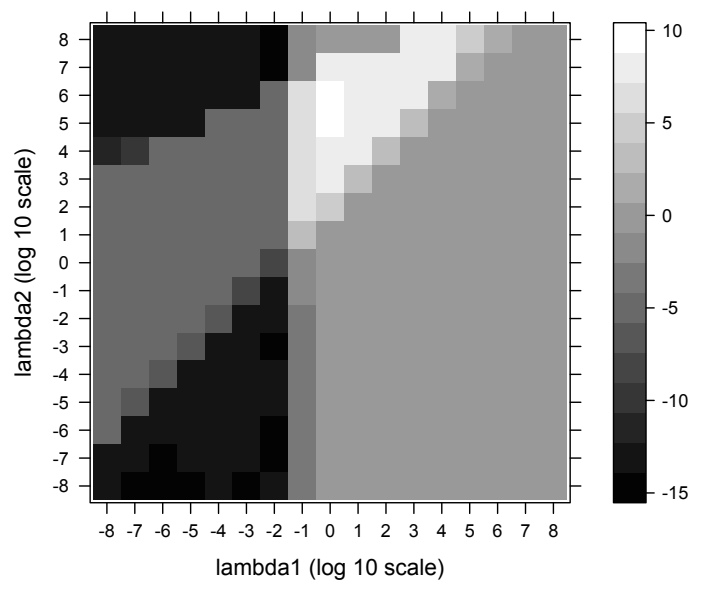

Figure 3: Difference of average computing time for the simulation described in section 5.2.3 based on 100 replications at each point in $\Lambda \times \Lambda$. Negative values represent where ridge fusion is faster than FGL. 
Table 7: Average CER reported with standard errors for the semi-supervised model based clustering simulation based on 50 independent replications.

\begin{tabular}{l|cc} 
& $p=50$ & $p=100$ \\
\hline Ridge & $0.01(0.01)$ & $0.07(0.04)$ \\
Ridge Labeled & $0.02(0.02)$ & $0.22(0.06)$ \\
FGL & $0.01(0.01)$ & $0.01(0.01)$ \\
FGL Labeled & $0.04(0.03)$ & $0.31(0.07)$
\end{tabular}

similar result to the other timing simulations in sections 5.2.1 and 5.2.2; ridge fusion is faster or comparable to FGL when $\lambda_{1}$ is small and otherwise FGL is faster. We find that on average over the entire grid that ridge fusion is on average 5 seconds faster. At the extremes, there was one point on the grid where ridge fusion was 595 times faster than FGL and another point on the grid where FGL was 322 times faster than ridge fusion.

\subsection{Regularization in semi-supervised model based clustering}

We evaluate the semi-supervised model based clustering methods proposed in section 4 by comparing the tuning parameter selection methods proposed in sections 4.3 and 2.3 . This simulation uses the same data generating model as that used in the simulation study in section 5.1.6 with $\rho=0.25$. Each replication will have 25 labeled and 250 unlabeled observations from each class. We compare the ridge fusion and FGL methods for semisupervised model based clustering on their ability to classify the unlabeled data for 50 independent replications when the tuning parameters are selected using the labeled data only via the methodology proposed in section 4.3. For each replication the QDA classification rule is formed by using the corresponding parameter estimates from the regularized semisupervised model based clustering. Results of this simulation are contained in Table 7 and show that using the method presented in section 4.3 to select the tuning parameter outperforms the method that ignores the unlabeled data. 
Table 8: Fraction of the validation data that is classified incorrectly for the Libra data example.

\begin{tabular}{l|c} 
& Fraction of Data Misclassified \\
\hline Ridge & $0 / 135$ \\
FGL & $9 / 135$ \\
RDA & $4 / 135$
\end{tabular}

\section{DATA EXAMPLE}

We compare ridge fusion, FGL and RDA on the Libras movement data from the UCI Machine Learning Repository, which describes the hand movements in Brazilian sign language (Bache and Lichman, 2013). The original data has 15 classes corresponding to the type of hand movements, with 90 variables that represent 45 different time points in a video that shows the hand movement. The variables represent where the hand is in the frame at a given time point. For this example we selected 3 classes that correspond to the movements of curved swing, horizontal swing, and vertical swing. We have taken 18 observations from each class for training while keeping 45 observations from each class for validation. Tuning parameters for each method were selected using 3 -fold validation likelihood due to the small sample size of the training set. The results of this analysis are reported in Table 8 , and show that the ridge fusion method outperforms FGL and RDA with regard to the number of observations classified incorrectly for the validation data.

We also apply the methodology from section 4 on the Libras movement data where the 45 validation points from each class are treated as the unlabeled data. Again we use a 3-fold validation likelihood based on the method proposed in section 4.3 . Table 9 contains the results. As we saw in the supervised case, the ridge fusion method has a smaller number of observations classified incorrectly when compared to FGL on the unlabeled data. 
Table 9: Fraction of the unlabeled data that is classified incorrectly using semi-supervised model based clustering methods for the Libra data example.

\begin{tabular}{l|c} 
& Fraction of Unlabeled Data Misclassified \\
\hline Ridge & $0 / 135$ \\
FGL & $5 / 135$
\end{tabular}

\section{ACKNOWLEDGMENTS}

This research is partially supported by the National Science Foundation grant DMS-1105650.

\section{References}

Bache, K. and Lichman, M. (2013), "UCI Machine Learning Repository," http://archive. ics.uci.edu/ml.

Basu, S., Banerjee, A., and Mooney, R. (2002), "Semi-supervised Clustering by Seeding," in Proceedings of the 19th International Conference on Machine Learning, 19-26.

Danaher, P. (2013), JGL: Performs the Joint Graphical Lasso for sparse inverse covariance estimation on multiple classes, URL http://CRAN.R-project.org/package=JGL, r package version 2.3 .

Danaher, P., Wang, P., and Witten, D. (2013), "The Joint Graphical Lasso for Inverse Covariance Estimation Across Multiple Classes," The Journal of Royal Statistical Society, Series B.

Dempster, A., Laird, N., and Rubin, D. (1977), "Maximum Likelihood From Incomplete Data via the EM Algorithm (with discussion)," Journal of The Royal Statistical Society, Series $B, 39,1-38$. 
Friedman, J. (1989), "Regularized Discriminant Analysis," Journal of the American Statistical Association, 84, 249-266.

Green, P. (1990), "On Use of the EM for Penalized Likelihood Estimation," Journal of the Royal Statistical Society, Series B, 52, 443-452.

Guo, J., Levina, E., Michailidis, G., and Zhu, J. (2011), "Joint Estimation of Multiple Graphical Models," Biometrika, 98, 1-15.

Huang, J., Liu, N., Pourahmadi, M., and Liu, L. (2006), "Covariance Matrix Selection and Estimation via Penalised Normal Likelihood," Biometrika, 93, 85-98.

Pourahmadi, M. (2011), "Covariance Estimation: The GLM and Regularization Perspective," Statistical Science, 26, 369-387.

Price, B. S. (2014), RidgeFusion: R Package for Ridge Fusion in Statistical Learning, R package version 1.0-3.

Rothman, A. J. and Forzani, L. (2013), "Properties of optimizations used in penalized Gaussian likelihood inverse covariance matrix estimation," Manuscript.

Ruan, L., Yuan, M., and Zou, H. (2011), "Regularized Parameter Estimation in HighDimensional Gaussian Mixture Models," Neural Computation, 23, 1605-1622.

Weihs, C., Ligges, U., Luebke, K., and Raabe, N. (2005), "klaR Analyzing German Business Cycles," in Data Analysis and Decision Support, eds. D. Baier, R. Decker, and L. SchmidtThieme, Berlin: Springer-Verlag, 335-343.

Witten, D. and Tibshirani, R. (2009), "Covariance Regularized Regression and Classification for High-Dimensional Problems," Journal of Royal Statistical Society, Series B, 71, 615636. 
Wu, C. J. (1983), "On the Convergence Properties of the EM Algorithm," Annals of Statistics, 11, 95-103.

Xie, B., Pan, W., and Shen, X. (2008), "Penalized Model Based Clustering with Cluster Specific Diagonal Covariance Matrices and Grouped Variables," Electronic Journal of Statistics, 2, 168-212.

Zhou, H., Pan, W., and Shen, X. (2009), "Penalized Model-Based Clustering with Unconstrained Covariance Matrices," Electronic Journal of Statistics, 3, 1473-1496. 